\title{
The Evasion of Antiviral Innate Immunity by Chicken DNA Viruses
}

\author{
Li Gao, Shijun Zheng and Yongqiang Wang* \\ Department of Preventive Veterinary Medicine, College of Veterinary Medicine, China Agricultural University, Beijing, China
}

The innate immune system constitutes the first line of host defense. Viruses have evolved multiple mechanisms to escape host immune surveillance, which has been explored extensively for human DNA viruses. There is growing evidence showing the interaction between avian DNA viruses and the host innate immune system. In this review, we will survey the present knowledge of chicken DNA viruses, then describe the functions of DNA sensors in avian innate immunity, and finally discuss recent progresses in chicken DNA virus evasion from host innate immune responses.

Keywords: chicken, DNA virus, PRRs, innate immunity, evasion

OPEN ACCESS

Edited by:

Chunfu Zheng,

University of Calgary, Canada

Reviewed by:

Yi-Quan Wu,

National Cancer Institute (NCl),

United States

Kai Li,

Harbin Veterinary Research Institute,

Chinese Academy of Agricultural

Sciences (CAAS), China

*Correspondence:

Yongqiang Wang

vetwyq@cau.edu.cn

Specialty section:

This article was submitted to

Virology,

a section of the journal

Frontiers in Microbiology

Received: 06 September 2021

Accepted: 11 October 2021

Published: 28 October 2021

Citation:

Gao L, Zheng S and Wang Y (2021)

The Evasion of Antiviral Innate Immunity by Chicken DNA Viruses.

Front. Microbiol. 12:771292.

doi: 10.3389/fmicb.2021.771292

\section{INTRODUCTION}

The innate immune system constitutes the first line of host defense. Upon virus infection, pathogen-associated molecular patterns (PAMPs), which are the components of foreign pathogens, will be recognized by germline-encoded pattern-recognition receptors (PRRs), leading to the initiation of innate antiviral immunity, such as the production of type I interferons (IFN-I) and pro-inflammatory cytokines. On the other hand, viruses have evolved multiple strategies to evade host immune surveillance which has been explored extensively for human viruses. There is growing evidence indicating the interaction between avian DNA viruses and the host's antiviral innate immunity. This review will focus on chicken DNA viruses and their evasion of host innate immune response, surveying the present knowledge of the function of DNA sensors in avian innate immunity, and discussing futuristic perspectives in this angle. Monumental reviews specific on DNA viruses and antiviral innate immunity have been summarized (Briard et al., 2020).

\section{THE MAINLY CIRCULATED CHICKEN DNA VIRUS}

Multiple DNA viruses threaten the poultry industry, including chicken infectious anemia virus (CIAV), Fowl adenovirus serotype 4 (FAdV-4), Marek's disease virus (MDV), infectious laryngotracheitis virus (ILTV), and fowl pox virus (FPV).

CIAV is a non-enveloped, single-stranded DNA virus, which belongs to the Gyrovirus genus, the Anelloviridae family. The genome of CIAV is a $2.3 \mathrm{~kb}$ covalently linked DNA ring coding for three viral proteins (Li et al., 2016). CIAV mainly infects bone marrow hemocytoblasts and thymus precursor lymphocytes (Miller et al., 2005). The severe damages caused by this virus such as weight loss, anemia, intramuscular hemorrhage, lymphoid atrophy, and bone marrow aplasia mostly appear among chicks less than 2-week-old and are void of maternally derived antibodies (Gimeno and Schat, 2018). Adult chickens are also infected horizontally 
with CIAV, though with subclinical symptoms after the maternal antibodies wane (Trinh et al., 2015; Fatoba and Adeleke, 2019).

CIAV infection causes chicken immunosuppression. Inoculation of 1-day-old chicks with CIAV suppresses functions of spleen and bone-marrow-derived macrophage, such as Fc receptor expression, phagocytosis, and IL-1 production (McConnell et al., 1993). Four days post inoculation of 1-dayold chicks with CIAV, the innate immune cytokine mRNA levels were analyzed using qRT-PCR and gene expression microarrays, the pro-inflammatory cytokines, including IL-1 $\beta$, IL-6, CXCLi2, and IFN-I, were inhibited across different tissues, except in the thymus (Giotis et al., 2015). Compared to the uninfected control thymus, CIAV infection-induced IL-1 $\beta$, IL-6, and IFN-I production by 3-4 folds. However, that is limited in magnitude. Not all of the pro-inflammatory cytokines could be induced by CIAV infection. For example, CXCLi2 was significantly inhibited in the bursa of the Fabricius at 11 days post-inoculation (Giotis et al., 2015). CIAV infection of MSB1 cells, a $\mathrm{T}$ cell line that supports CIAV replication in vitro, cannot induce an effective antiviral innate immune response either. When MSB-1 cells were infected with CIAV, IFN-I and interferon-stimulated genes (ISGs) mRNAs were only slightly induced at 24 and $48 \mathrm{hpi}$, and even declined at $72 \mathrm{hpi}$ compared with uninfected control cells (Giotis et al., 2015, 2018).

FAdV belongs to the genus Aviadenovirus and the family Adenoviridae. FAdV can be divided into five species (FAdV-A to FAdV-E) according to their restriction enzyme digestion pattern (Zsak and Kisary, 1984; Li et al., 2017) and 12 serotypes (FAdV-1-8a and 8b-11) based on cross-neutralization test (Hess, 2000; Kim et al., 2014). FAdV-4, one of 12 FAdV serotypes, has been grouped into FAdV-C (Li et al., 2017; Pan et al., 2017). The genome of FAdV-4 is an $\sim 43-46 \mathrm{~kb}$ double-stranded DNA, encoding for 10 major structural proteins in the viral particles and 11 non-structural proteins, which participate in viral replication and pathogenesis ( $\mathrm{Li}$ et al., 2017). FAdV-4 mainly infects chicken livers and can cause severe damage in chickens, especially for broilers aged 3- to 5-week-old. The mortality rate caused by FAdV-4 infection is about $30-70 \%$ (Mansoor et al., 2011; Shah et al., 2012; Zhao et al., 2015; Shah et al., 2016). FAdV-4 infection in chickens can induce hydropericardium syndrome (HPS), which is a severe disease characterized by the accumulation of clear, straw-colored fluid in the pericardial sac, as well as nephritis and hepatitis (AbdulAziz and Al-Attar, 1991; Abdul-Aziz and Hasan, 1995; Abe et al., 1998).

A previous review has summarized that human adenovirus (HAdV) inhibits innate host immunity through its early gene products and is implicated in pathogenesis (Wang and Zhao, 2019). For example, the E1A oncogene of HAdV-5 blocks the cellular response to IFNs (Ackrill et al., 1991) and attenuates the transcription of IL-6 (Janaswami et al., 1992). Hyper-virulent FAdV-4 also suppresses host immune response. Compared to the mock-infected control, FAdV-4 infection depleted lymphocytes in the thymus and bursa through apoptosis and severe inflammatory response (Niu et al., 2019b). Analysis of the mRNA expression in livers of FAdV-4-infected chickens identified that IFN was downregulated at 7 days post-infection
(Ren et al., 2019). However, the mechanisms of how FAdV-4 suppresses host innate immune response remain to be explored.

Marek's disease virus (MDV) is a member of the genus Mardivirus, which belongs to the subfamily Alphaherpesvirinae in the order of Herpesvirales (Gimeno and Schat, 2018). Despite the use of vaccines, MDV infection still circulates in poultry flocks. It mainly infects chickens, causing lymphomas and different kinds of nonneoplastic syndromes, including neurological syndromes, lymphodegenerative syndrome, and atherosclerosis (Gimeno and Schat, 2018; Bertzbach et al., 2020).

As an immunosuppressive virus, MDV infection inhibits the host's innate immune responses through multiple approaches. RT-PCR and ELISA analysis suggested that, IFN-I mRNA and protein expressions were significantly down-regulated in the thymus and bursa of MDV-infected groups (Sun et al., 2019). Furthermore, the potential of MDV inhibition on IFN-I was associated with their pathogenesis (Sun et al., 2019). Macrophages and Natural killer (NK) cells are important components of the innate immune system. It has been investigated that MDV efficiently infects NK cells (Bertzbach et al., 2019) and macrophages (Barrow et al., 2003). However, how MDV infection of macrophages and NK cells influences the innate and subsequent adaptive immune response remains further to be investigated.

ILTV is classified in the genus Iltovirus, subfamily Alphaherpesvirinae within Herpesviridae family (Gowthaman et al., 2020). The double strand DNA genome of ILTV is about $150 \mathrm{~kb}$, containing short and long unique regions $\left(\mathrm{U}_{\mathrm{S}}\right.$, $\mathrm{U}_{\mathrm{L}}$ ), and inverted repeats (IR, TR) flanking the $\mathrm{U}_{S}$ region (Fuchs et al., 2007; Gowthaman et al., 2020). ILTV mainly infects the upper respiratory tract of chickens and causes different clinical symptoms depending on the virulence of the infected strains. The mortality rates caused by ILTV infection vary from 0 to more than 70\% (Ou and Giambrone, 2012). Live attenuated vaccines have been applied to prevent ILTV infection and proved to be efficacious (Garcia and Zavala, 2019). However, similar to other live virus vaccines, vaccination with attenuated ILTV strains also faces the risk caused by the remaining virulence.

ILTV infection destroys the respiratory epithelium and induces the infiltration of inflammatory cells (Gowthaman et al., 2020). Inflammatory responses are essential in the innate immune response against ILTV infection. An in vitro analysis reported that ILTV infection upregulated many genes associated with inflammatory response, including IL-6 and IL-8 (Coppo et al., 2013). Further studies have identified glycoprotein G $(\mathrm{gG})$, a protein conserved among alphaherpesviruses, as a virulence factor that can inhibit cytokine and chemokine transcriptions, thus interfering with the antiviral inflammatory responses (Coppo et al., 2018). gG also influences the composition of inflammatory cell infiltration in the tracheal mucosa (Coppo et al., 2018). However, more details about how ILTV evades host innate immune responses remain unknown.

FPV belongs to the Avipoxvirus genus, Poxviridae family. As a prototypic species of poxviruses, FPV is a large enveloped virus containing double-stranded DNA genome (Giotis and Skinner, 2019). FPV infects domestic chickens and gallinaceous birds, causing diseases mainly presented as diphtheritic and 
cutaneous forms (Giotis and Skinner, 2019). Vaccination has been applied to prevent FPV infection (Bhanuprakash et al., 2012). However, in regions where biting insects are prevalent, fowl pox still threats the poultry industry.

Like the other poxvirus, FPV has been extensively used as vaccine vectors for different poultry and human diseases (Skinner et al., 2005). FPV infection inhibits host innate immune response, especially IFN-I response. It has been investigated that $f p v 012$ and $f p v 184$ genes of FPV block the expression of IFN-I (Laidlaw et al., 2013; Giotis et al., 2020), while fpv014 gene contributes to the evasion of FPV from the antiviral effect of IFN-I (Buttigieg et al., 2013).

\section{chcGAS}

cGAS, which belongs to the nucleotidyltransferase family (Sun et al., 2013), expresses and functions in multiple species (Wang et al., 2015; Li et al., 2020; Qiao et al., 2021). cGAS binds DNA through both the C-terminal NTase domain and N-terminal domain in a sequence-independent manner (Civril et al., 2013; Gao et al., 2013; Kranzusch et al., 2013), and both foreign and host DNA can activate cGAS. cGAS binding to dsDNA undergoes conformational changes and then initiates the synthesis of the nucleotide 2'3'-cGAMP from ATP and GTP (Ablasser et al., 2013; Li et al., 2013a; Andreeva et al., 2017; Hall et al., 2017; Du and Chen, 2018). 2'3'-cGAMP is a second messenger bound by the Stimulator of Interferon Genes (STING; Ablasser et al., 2013; Zhang et al., 2013), a signaling adapter at the endoplasmic reticulum (ER). The binding of cGAMP activates STING, followed by translocating to the Golgi and activating downstream pathways to induce the IFN-I and inflammatory cytokine response (Ouyang et al., 2012; Shang et al., 2012, 2019; Zhang et al., 2019). Infections with cGAS-deficient cells and mice have indicated that the cGAS-STING pathway is indispensable for host immune response against DNA virus infections (Li et al., 2013b; Schoggins et al., 2014).

cGAS also functions in chickens (Vitak et al., 2016; Li et al., 2020; Neerukonda and Katneni, 2020). There is 438 aa in Chicken cGAS (chcGAS), and its predicted structure is highly similar to that of human cGAS. The homology of chcGAS with human cGAS is $48.5 \%$ ( $\mathrm{Li}$ et al., 2020). The chcGAS mRNAs are expressed in various chicken tissues, such as the bursa of Fabricius, thymus, spleen and lung, with the highest in bone marrow (Wang et al., 2020). Subcellular localization assay has indicated that chcGAS does not localize in the mitochondria, lysosomes or Golgi apparatus, while it distributes in the cytosol and is partially co-localized with the endoplasmic reticulum (ER; Wang et al., 2020).

chcGAS responses to exogenous DNAs derived from virus infection or dsDNA transfection, and endogenous dsDNA upon DNA damages (Li et al., 2020). Overexpression of chcGAS induced IFN- $\beta$ and IL- $1 \beta$ mRNA expression in a way dependent on chSTING, chTBK1 and chIRF7 (Wang et al., 2020). Transfection of poly(dA:dT) and HS-DNA potently promoted the induction of IFN- $\beta$ and IL-1 $\beta$ by chcGAS (Li et al., 2020). Knockout of chcGAS reduced the IFN- $\beta$ and IL- $1 \beta$ mRNA expression induced by DNA virus infections, such as HSV-1 and FAdV-4, and subsequently enhanced virus replication (Wang et al., 2020).

\section{chSTING}

STING has been identified as an essential adaptor that facilitates innate immune signaling against DNA and DNA virus infections (Ishikawa and Barber, 2008; Zhong et al., 2008). Further studies indicated that STING functions as an indispensable adaptor downstream of multiple DNA sensors (Unterholzner et al., 2010; Zhang et al., 2011; Ferguson et al., 2012; Dunphy et al., 2018; Wang et al., 2019; Li et al., 2019b).

There is 379 aa in chicken STING (chSTING). As predicted, chSTING contains the TMEM173 domain (aa 50-342), which is highly conserved (Ran et al., 2018; Li et al., 2020). Sequence alignment showed that chSTING has about $43 \%$ identities in aa to human STING (Cheng et al., 2015; Li et al., 2020). chSTING mRNAs are broadly expressed in chicken tissues, especially with the highest levels in the spleen (Cheng et al., 2015). Similar to its human homolog, chSTING is also predominantly localized in the outer membrane of the ER as well as the mitochondrial membrane (Cheng et al., 2015).

chSTING has an essential role in initiating innate immune responses against virus infections in chickens. Overexpression of chSTING in DF-1 cells activated IRF-7 and NF- $\kappa$ B to trigger the expression of IFN- $\beta$ as well as IFN stimulated genes (ISGs; Cheng et al., 2015). Pro-inflammatory cytokine mRNAs are also upregulated upon chSTING overexpression (Cheng et al., 2015). Moreover, transfection of chSTING into DF-1 cells suppressed AIV and NDV replications, while knockdown of endogenous chSTING inhibited poly(dA:dT)-, and poly(I:C)triggered IFN- $\beta$ induction (Cheng et al., 2015). Further studies have shown that chSTING is an essential adaptor in innate immune responses. chSTING mediates the IFN-stimulating signals triggered by different DNA and RNA sensors, including chMDA5 (Cheng et al., 2015), chcGAS (Li et al., 2020), chDDX3X (Niu et al., 2019a), and chDDX41 (Cheng et al., 2017). Endogenous Co-IP and LC-MS/MS suggested that, as in mammalian cells, chSTING interacted with chTBK1 to stimulate IFN-I responses (Cheng et al., 2018).

However, many important issues have not been clarified, such as the features of dsDNA bound by chcGAS, the structures, and mechanisms employed by chcGAS, and chSTING to deliver signals and the regulation of chcGAS-STING pathways.

\section{chDDX41}

RNA helicases are another group of sensors that participate in DNA-induced IFN-I response. RNA helicases comprise two subgroups: the DEAD-box helicases (DDX) and the DEAH-box helicases (DHX; Briard et al., 2020).

DDX41, which belongs to the DEXDc family of helicases, has been identified as a DNA sensor in DCs in humans and mice (Zhang et al., 2011). chDDX41 has also been cloned and 
analyzed (Cheng et al., 2017). Sequence analysis has indicated $1,815 \mathrm{bp}$ in chDDX41, encoding for 604 aa residues, which shares 93.7\% identity with human DDX41 (Cheng et al., 2017). chDDX41 distributes widely in multiple tissues, especially with the highest expression in the crop (Cheng et al., 2017). Although the other members of the DExD family have been identified as IFN-stimulated genes, the expression of chDDX41 is not upregulated by IFN- $\beta$. Like human DDX41, chDDX41 is essential for the IFN $-\beta$ induction in response to dsDNA poly(dA:dT) transfection and DNA virus infection (Cheng et al., 2017).

Human DHX36 and DHX9, members of the DHX helicases, besides their RNA-sensing function, also have a role in sensing CpG DNA and inducing IFN-I and pro-inflammatory cytokine responses via IRF7 and NF-KB pathways (Kim et al., 2010). DHX9 binds CpG-B by the DUF Domain, while DHX36 binds CpG-A through the DEAH Domain (Kim et al., 2010). Knockdown of DHX36 or DHX9 potently reduced the IFN and pro-inflammatory cytokine expressions in pDCs infected with a DNA virus while had no impact on an RNA virus infection (Kim et al., 2010).

An evolutionary analysis of the DEAD-box helicase family has identified that DHX9 is genetically deficient in chickens while DHX36 is conserved (Sato et al., 2015). Thus far, the DNA sensing and antiviral function of DHX36 in chickens remain to be explored.

\section{chTLR21}

As a group of evolutionarily conserved molecules, Toll-like receptors (TLRs) sense different PAMPs. Of them, Toll-like receptor 9 (TLR9) residues in endosomes for sensing DNA. The structure of TLR9 comprises a Toll/IL-1 receptor (TIR) domain and a leucine-rich repeat (LRR) domain (Briard et al., 2020). Upon binding to unmethylated CpG DNA, TLR9 dimerizes and recruits the adaptor protein MyD88, leading to the IFN-I and inflammatory cytokine responses through the transcription factor NF-kB, IRF7, or IRF1 (Briard et al., 2020).

However, the counterpart of mammalian TLR9 does not exist in the chicken genome. Instead, there is a functional homolog to mammalian TLR9 in the recognition of CpG oligodeoxynucleotides (ODNs), that is, chicken TLR21 (chTLR21), a protein of 972 aa residues (Brownlie et al., 2009; Keestra et al., 2010). It has been predicted that chTLR21 has three domains: a cytoplasmic (TIR) domain, a single transmembrane region, and an extracellular domain containing 27 LRRs with cysteine-rich capping structures at both ends (Keestra et al., 2010). Compared to TLR21 of other species, chTLR21 has high similarities with TLR21 of Xenopus tropicalis (61\%) and Takifugu rubripes (57\%) and appears more distantly to murine TLR13 (47\%; Chuang et al., 2020). As for hTLR9, there is only $38 \%$ similarity in aa between chTLR 21 and hTLR9, while two aa residues, which are essential for the interaction between CpG DNA and TLR9 (Asp534 and Tyr536), appear to be conserved in chTLR21 (Rutz et al., 2004; Wei et al., 2009). Cellular distribution analysis using confocal laser microscopy on transfected HEK293 cells has suggested that the co-expressed
chTLR21-FLAG and hTLR9 have similar localization profiles inside the cells (Chuang et al., 2020). chTLR21 is mainly expressed in immune-relevant tissues. The bursa of the Fabricius and spleen express the highest level of chTLR21, while the lung and thymus have a modest expression (Chuang et al., 2020). chTLR21 expression could be induced by stimulation with CpG-ODN. Inoculation of class B CpG ODN subcutaneously potently upregulated the expression of TLR21, interleukin (IL)- $1 \beta$, and interferon (IFN)- $\gamma$ mRNA in the blood cells (Chrzastek and Wieliczko, 2014). Another study conducted in vitro indicated that the mRNA expression levels of TLR21 and IFN- $\gamma$ were substantially increased by Class B CpG ODN stimulation at $1 \mathrm{~h}$ post-stimulation in a concentration-dependent manner, while this phenomenon only exited in HG cells isolated from young birds (Chrzastek et al., 2014). chTLR21 expression could also be induced by poly (I:C), a dsRNA mimics stimulating TLR3 signaling pathways, in DF-1 cells (Jang and Song, 2020).

Although chTLR21 acts as a functional homolog of mammalian TLR9, there are differences between chTLR21 and hTLR9 in ligand specificities. For example, hTLR9 prefers the CpG-DNA hexameric motifs GTCGTT, while various CpG-ODNs hexamer motifs can activate chTLR21 (Keestra et al., 2010; Chuang et al., 2020). A recent study further revealed that CpG-ODNs with lengths between 15 and 31 nucleotides and different spaces between CpG-hexamer motifs could be sensed by chTLR21 (Chuang et al., 2020).

Stimulation of HEK293 cells in which chTLR21 was transfected with CpG-ODNs resulted in NF- $\mathrm{KB}$ activation and subsequently IL-1 $\beta$ and IL- 6 mRNA expressions. Using RNA interference (RNAi) technology has confirmed that MyD88 is dependent on TLR21 in response to CpG-ODN in HD11 macrophages (Keestra et al., 2010; Chuang et al., 2020). However, the exact molecular mechanism of chTLR21 activation and signal transduction remains to be explored further.

It has been reported that chTLR21 has been involved in host antiviral responses recently. CpG-ODN, the TLR21 agonist, significantly increased the IL-2, IFN- $\gamma$, IL-1, and IL-4 levels in the peripheral blood mononuclear cells (PBMCs) of chickens which responded to IBDV infection and greatly reduced the mortality rate of vvIBDV-infected chicks compared with the birds in the control group (Sachan et al., 2019). Treating the 9-11day-old SPF embryonated chicken eggs (ECEs) with CpG-ODN induced ISGs, IL-1 $\beta$, and IFN- $\gamma v i z$. OAS and iNOS genes in CAM. Furthermore, co-inoculation of TLR21 ligand CpG-ODN and avian IBV significantly reduced virus titers (Sharma et al., 2020). Besides, goose TLR21, which has homology to chTLR21, has been reported to have a role in sensing invasive DNA viruses. TLR21, IFN-I, pro-inflammatory cytokines (IL-6 and IL-1 $\beta$ ) were potently induced in PBMCs challenged with agonist ODN2006 and new type gosling viral enteritis virus (NGVEV; Qi et al., 2016). TLR21 also has a role in duck immune response against DNA and DNA viruses. Stimulation of DEFs transfected with duTLR21 with CpG-ODN activated NF-кB, followed by the increased expression of the IFN- $\alpha$, IL- 6 , and IL- $1 \beta$ mRNA, whereas knockdown of duTLR21 impaired the expression of these genes (Cheng et al., 2019; Chuang et al., 2020). Furthermore, an in vitro study has indicated that ectopic expression of 
duTLR21 blocks the duck plague virus (DPV) replication, while knockdown of duTLR21 efficiently facilitates DPV replication (Cheng et al., 2019).

\section{CHICKEN DNA VIRUS' EVASION FROM DNA SENSOR-INDUCED ANTIVIRAL RESPONSE}

Chicken DNA viruses have evolved various mechanisms to inhibit the chcGAS-STING signaling pathway to achieve a successful infection. MDV is a highly pathogenic and oncogenic chicken herpesvirus. MDV infection in chickens induces immunosuppression and fatal $\mathrm{T}$ cell lymphomas. To achieve a persistent infection, MDV suppresses host innate immune responses. Knock-down of cGAS or STING in cells has shown that cGAS-STING pathway has an important role in the inducing IFN-response against MDV infection (Gao et al., 2019). Further studies have revealed that multiple MDV proteins inhibit IFN-response through different mechanisms (Figure 1; Gao et al., 2019; Li et al., 2019a;
Liu et al., 2019). VP23 is an integral capsid protein of herpesvirus, essential for capsid assembly and viral replication (Kut and Rasschaert, 2004). Besides, VP23 is also essential in regulating host antiviral immunity via inhibiting IFN-I induction activated by cGAS-STING. Overexpression of VP23 potently attenuated the induction of IFN-I upon viral infection and enhanced viral replication in chicken fibroblast (DF-1) and chicken macrophage (HD11) cell lines, while VP23 knockdown during MDV infection promoted the IFN-response and inhibited MDV replication (Gao et al., 2019). Dualluciferase reporter assay has suggested that VP23 inhibits the activation of IRF7 while does not affect NF- $\kappa$ B. By interacting with the same region of IRF7 as TBK1, VP23 also disrupted the TBK1-IRF7 association, thereby preventing IRF7 activation and inhibiting IFN-induction in VP23transfected cells (Gao et al., 2019). Meq is a major oncoprotein of MDV and has an essential role in counteracting host innate immune response (Li et al., 2019a). Meq can prevent the associations of STING-TBK1 and STING-IRF7 by interacting with STING and IRF7, thereby suppressing IRF7 activation and IFN- $\beta$ response. Moreover, overexpression of

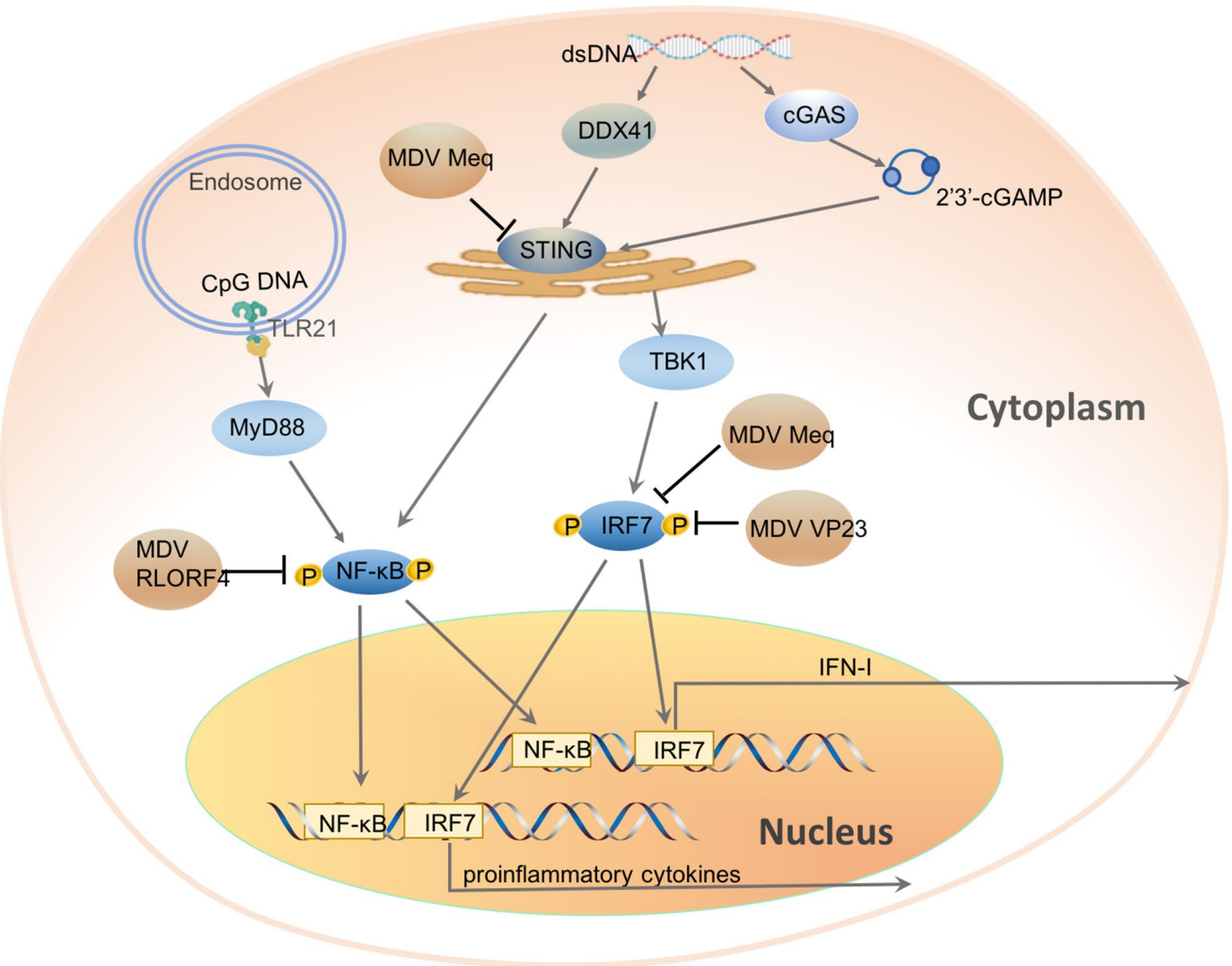

FIGURE 1 | Chicken DNA viruses' evasion of DNA sensor-induced antiviral innate immunity. cGAS and DDX41 localize in the cytoplasm and sense cytosolic dsDNA directly, DNA recognition induces cGAS and DDX41 activation. Upon activation, cGAS catalyzes the synthesis of the nucleotide 2'3'-cGAMP from ATP and GTP, which will be bound by STING and induce STING activation, while DDX41 interacts and activates STING directly. STING activation recruits and activates TBK1, followed by phosphorylation and nuclear translocation of transcription factor IRF7, which initiates transcription of IFN-I and pro-inflammatory cytokines. STING also activates NF-kB. TLR21, which resides in the endosome, senses CpG DNA and activates NF-kB through adaptor protein MyD88. MDV encoded proteins Meq interacts with STING and IRF7, VP23 interacts with IRF7, while RLORF4 binds the endogenous NF-kB. 
Meq selectively suppresses the expression of IFN-I and downstream antiviral genes upon DNA virus infection and cytosolic dsDNA stimulation. In contrast, MDV with Meq-deficiency significantly induced higher levels of IFN- $\beta$ and downstream IFN-stimulated genes, resulting in attenuated viral replication and transformation in CEFs and chickens. Being different from VP23 and Meq, MDV RLORF4 selectively inhibits cGAS-STING-mediated NF- $\mathrm{B}$ activation but not IRF7. RLORF4 is a 142-amino-acid protein encoded by a single transcript in wild-type strains. Attenuation of MDV via serial passages in vitro has indicated that RLORF4 is essential for MDV pathogensis. Deletion of RLORF4 from virulent MDV significantly inhibited viral pathogenicity in vivo (Jarosinski et al., 2005, 2015; Jarosinski and Schat, 2007; Vega-Rodriguez et al., 2019). Further studies have indicated that ectopically expressed RLORF4 suppresses the activation of IFN- $\beta$ promoter stimulated by cGAS and STING, while knockout of RLORF4 from the MDV genome enhances IL-6 and IFN- $\beta$ productions in vivo and in vitro. By interacting with NF- $\mathrm{B}$ subunits p65 and p50, RLORF4 could prevent the nuclear translocation of p65 and p50 induced by interferonstimulatory DNA and TNF- $\alpha$, thereby inhibiting IFN- $\beta$ responses (Liu et al., 2019). However, as an oncogenic herpesvirus, MDV encodes multiple proteins which have different roles in viral replications (Davidson, 2020). Whether other proteins have effects on the DNA sensing pathway remains to be investigated.

Nevertheless, how other chicken DNA viruses, such as chicken infectious anemia virus (CIAV), fowl adenovirus (FAdV), and infectious laryngotracheitis virus (ILTV), evade cGASSTING mediated antiviral signaling is still obscure.

\section{REFERENCES}

Abdul-Aziz, T. A., and Al-Attar, M. A. (1991). New syndrome in Iraqi chicks. Vet. Rec. 129:272. doi: 10.1136/vr.129.12.272

Abdul-Aziz, T. A., and Hasan, S. Y. (1995). Hydropericardium syndrome in broiler chickens: its contagious nature and pathology. Res. Vet. Sci. 59, 219-221. doi: 10.1016/0034-5288(95)90005-5

Abe, T., Nakamura, K., Tojo, H., Mase, M., Shibahara, T., Yamaguchi, S., et al. (1998). Histology, immunohistochemistry, and ultrastructure of hydropericardium syndrome in adult broiler breeders and broiler chicks. Avian Dis. 42, 606-612. doi: 10.2307/1592690

Ablasser, A., Goldeck, M., Cavlar, T., Deimling, T., Witte, G., Rohl, I., et al. (2013). cGAS produces a 2'-5'-linked cyclic dinucleotide second messenger that activates STING. Nature 498, 380-384. doi: 10.1038/nature12306

Ackrill, A. M., Foster, G. R., Laxton, C. D., Flavell, D. M., Stark, G. R., and Kerr, I. M. (1991). Inhibition of the cellular response to interferons by products of the adenovirus type 5 E1A oncogene. Nucleic Acids Res. 19, 4387-4393. doi: 10.1093/nar/19.16.4387

Andreeva, L., Hiller, B., Kostrewa, D., Lassig, C., De Oliveira Mann, C. C., Jan Drexler, D., et al. (2017). cGAS senses long and HMGB/TFAM-bound U-turn DNA by forming protein-DNA ladders. Nature 549, 394-398. doi: $10.1038 /$ nature 23890

Barrow, A. D., Burgess, S. C., Baigent, S. J., Howes, K., and Nair, V. K. (2003). Infection of macrophages by a lymphotropic herpesvirus: a new tropism for Marek's disease virus. J. Gen. Virol. 84, 2635-2645. doi: 10.1099/vir.0.19206-0

Bertzbach, L. D., Conradie, A. M., You, Y., and Kaufer, B. B. (2020). Latest insights into Marek's disease virus pathogenesis and tumorigenesis. Cancer 12:647. doi: $10.3390 /$ cancers 12030647
However, there is limited information on how chicken DNA viruses evade other DNA sensors to induce antiviral innate immune responses, which requires further investigation.

\section{FUTURE PERSPECTIVES}

The ability of viruses to modulate host antiviral innate immunity is crucial for successful infections. Viruses can adopt multiple strategies to escape host immune surveillance, such as avoiding the exposure of the genome to DNA sensors, inhibiting the synthesis of the second messenger, and blocking the activation of signaling adaptors. Great gaps still exist in understanding how chicken DNA viruses inhibit host innate immunity and get infections, which blocks the design of a new generation of effective vaccines.

\section{AUTHOR CONTRIBUTIONS}

YW and LG conceptualized the mini-review topic. LG and YW contributed to writing the review. LG, SZ, and YW contributed to editing the review. All authors contributed to the article and approved the submitted version.

\section{FUNDING}

This work was supported by grants from the National Natural Science Foundation of China (\# 32072850) and Earmarked Fund for Modern Agro-Industry Technology Research System (\#CARS-40), China.

Bertzbach, L. D., Van Haarlem, D. A., Hartle, S., Kaufer, B. B., and Jansen, C. A (2019). Marek's disease virus infection of natural killer cells. Microorganisms 7:588. doi: 10.3390/microorganisms7120588

Bhanuprakash, V., Hosamani, M., Venkatesan, G., Balamurugan, V., Yogisharadhya, R., and Singh, R. K. (2012). Animal poxvirus vaccines: a comprehensive review. Expert Rev. Vaccines 11, 1355-1374. doi: 10.1586/ erv.12.116

Briard, B., Place, D. E., and Kanneganti, T. D. (2020). DNA sensing in the innate immune response. Physiology 35, 112-124. doi: 10.1152/ physiol.00022.2019

Brownlie, R., Zhu, J., Allan, B., Mutwiri, G. K., Babiuk, L. A., Potter, A., et al. (2009). Chicken TLR21 acts as a functional homologue to mammalian TLR9 in the recognition of CpG oligodeoxynucleotides. Mol. Immunol. 46, 3163-3170. doi: 10.1016/j.molimm.2009.06.002

Buttigieg, K., Laidlaw, S. M., Ross, C., Davies, M., Goodbourn, S., and Skinner, M. A. (2013). Genetic screen of a library of chimeric poxviruses identifies an ankyrin repeat protein involved in resistance to the avian type I interferon response. J. Virol. 87, 5028-5040. doi: 10.1128/JVI.02738-12

Cheng, Y., Liu, Y., Wang, Y., Niu, Q., Gao, Q., Fu, Q., et al. (2017). Chicken DNA virus sensor DDX41 activates IFN-beta signaling pathway dependent on STING. Dev. Comp. Immunol. 76, 334-342. doi: 10.1016/j.dci.2017. 07.001

Cheng, Y., Lun, M., Liu, Y., Wang, H., Yan, Y., and Sun, J. (2018). CRISPR/ Cas9-mediated chicken TBK1 gene knockout and its essential role in STINGmediated IFN-beta induction in chicken cells. Front. Immunol. 9:3010. doi: 10.3389/fimmu.2018.03010

Cheng, Y., Sun, Y., Wang, H., Yan, Y., Ding, C., and Sun, J. (2015). Chicken STING mediates activation of the IFN gene independently of the RIG-I gene. J. Immunol. 195, 3922-3936. doi: 10.4049/jimmunol.1500638 
Cheng, D., Wu, X., Jia, R., Wang, M., Chen, S., Liu, M., et al. (2019). CpG oligodeoxynucleotide-specific duck TLR21 mediates activation of NF-kappaB signaling pathway and plays an important role in the host defence of DPV infection. Mol. Immunol. 106, 87-98. doi: 10.1016/j.molimm.2018. 12.019

Chrzastek, K., Borowska, D., Kaiser, P., and Vervelde, L. (2014). Class B CpG ODN stimulation upregulates expression of TLR21 and IFN-gamma in chicken Harderian gland cells. Vet. Immunol. Immunopathol. 160, 293-299. doi: 10.1016/j.vetimm.2014.04.010

Chrzastek, K., and Wieliczko, A. (2014). The effects of subcutaneous and intraocular administration of class B ODN CpG in chicken on the expression of TLR21, IFN-gamma and IL-1beta. Pol. J. Vet. Sci. 17, 593-599. doi: 10.2478/pjvs-2014-0089

Chuang, Y. C., Tseng, J. C., Yang, J. X., Liu, Y. L., Yeh, D. W., Lai, C. Y., et al. (2020). Toll-like receptor 21 of chicken and duck recognize a broad array of immunostimulatory CpG-oligodeoxynucleotide sequences. Vaccine 8:639. doi: 10.3390/vaccines8040639

Civril, F., Deimling, T., De Oliveira Mann, C. C., Ablasser, A., Moldt, M., Witte, G., et al. (2013). Structural mechanism of cytosolic DNA sensing by cGAS. Nature 498, 332-337. doi: 10.1038/nature12305

Coppo, M. J. C., Devlin, J. M., Legione, A. R., Vaz, P. K., Lee, S. W., Quinteros, J. A., et al. (2018). Infectious laryngotracheitis virus viral chemokine-binding protein glycoprotein $G$ alters transcription of key inflammatory mediators in vitro and in vivo. J. Virol. 92, e01534-e01617. doi: 10.1128/JVI.01534-17

Coppo, M. J., Hartley, C. A., and Devlin, J. M. (2013). Immune responses to infectious laryngotracheitis virus. Dev. Comp. Immunol. 41, 454-462. doi: 10.1016/j.dci.2013.03.022

Davidson, I. (2020). Out of sight, but not out of mind: aspects of the avian oncogenic herpesvirus, Marek's disease virus. Animals 10:1319. doi: 10.3390/ ani10081319

Du, M., and Chen, Z. J. (2018). DNA-induced liquid phase condensation of cGAS activates innate immune signaling. Science 361, 704-709. doi: 10.1126/ science.aat 1022

Dunphy, G., Flannery, S. M., Almine, J. F., Connolly, D. J., Paulus, C., Jonsson, K. L., et al. (2018). Non-canonical activation of the DNA sensing adaptor STING by ATM and IFI16 mediates NF-kappaB signaling after nuclear DNA damage. Mol. Cell 71, 745.e745-760.e745. doi: 10.1016/j.molcel.2018.07.034

Fatoba, A. J., and Adeleke, M. A. (2019). Chicken anemia virus: a deadly pathogen of poultry. Acta Virol. 63, 19-25. doi: 10.4149/av_2019_110

Ferguson, B. J., Mansur, D. S., Peters, N. E., Ren, H., and Smith, G. L. (2012). DNA-PK is a DNA sensor for IRF-3-dependent innate immunity. elife 1:e00047. doi: 10.7554/eLife.00047

Fuchs, W., Veits, J., Helferich, D., Granzow, H., Teifke, J. P., and Mettenleiter, T. C. (2007). Molecular biology of avian infectious laryngotracheitis virus. Vet. Res. 38, 261-279. doi: 10.1051/vetres:200657

Gao, L., Li, K., Zhang, Y., Liu, Y., Liu, C., Zhang, Y., et al. (2019). Inhibition of DNA-sensing pathway by Marek's disease virus VP23 protein through suppression of interferon regulatory factor 7 activation. J. Virol. 93, e01934e00218. doi: 10.1128/JVI.01934-18

Gao, D., Wu, J., Wu, Y. T., Du, F., Aroh, C., Yan, N., et al. (2013). Cyclic GMP-AMP synthase is an innate immune sensor of HIV and other retroviruses. Science 341, 903-906. doi: 10.1126/science.1240933

Garcia, M., and Zavala, G. (2019). Commercial vaccines and vaccination strategies against infectious laryngotracheitis: what we have learned and knowledge gaps that remain. Avian Dis. 63, 325-334. doi: 10.1637/11967-090218-Review.1

Gimeno, I. M., and Schat, K. A. (2018). Virus-induced immunosuppression in chickens. Avian Dis. 62, 272-285. doi: 10.1637/11841-041318-Review.1

Giotis, E. S., Laidlaw, S. M., Bidgood, S. R., Albrecht, D., Burden, J. J., Robey, R. C., et al. (2020). Modulation of early host innate immune response by an avipox vaccine virus' lateral body protein. Biomedicine 8:634. doi: 10.3390/biomedicines 8120634

Giotis, E. S., Rothwell, L., Scott, A., Hu, T., Talbot, R., Todd, D., et al. (2015). Transcriptomic profiling of virus-host cell interactions following chicken anaemia virus (CAV) infection in an in vivo model. PLoS One 10:e134866. doi: 10.1371/journal.pone.0134866

Giotis, E. S., Scott, A., Rothwell, L., Hu, T., Talbot, R., Todd, D., et al. (2018). Chicken anaemia virus evades host immune responses in transformed lymphocytes. J. Gen. Virol. 99, 321-327. doi: 10.1099/jgv.0.001011
Giotis, E. S., and Skinner, M. A. (2019). Spotlight on avian pathology: fowlpox virus. Avian Pathol. 48, 87-90. doi: 10.1080/03079457.2018.1554893

Gowthaman, V., Kumar, S., Koul, M., Dave, U., Murthy, T., Munuswamy, P., et al. (2020). Infectious laryngotracheitis: etiology, epidemiology, pathobiology, and advances in diagnosis and control: a comprehensive review. Vet. Q. 40, 140-161. doi: 10.1080/01652176.2020.1759845

Hall, J., Ralph, E. C., Shanker, S., Wang, H., Byrnes, L. J., Horst, R., et al. (2017). The catalytic mechanism of cyclic GMP-AMP synthase (cGAS) and implications for innate immunity and inhibition. Protein Sci. 26, 2367-2380. doi: $10.1002 /$ pro.3304

Hess, M. (2000). Detection and differentiation of avian adenoviruses: a review. Avian Pathol. 29, 195-206. doi: 10.1080/03079450050045440

Ishikawa, H., and Barber, G. N. (2008). STING is an endoplasmic reticulum adaptor that facilitates innate immune signalling. Nature 455, 674-678. doi: $10.1038 /$ nature 07317

Janaswami, P. M., Kalvakolanu, D. V., Zhang, Y., and Sen, G. C. (1992). Transcriptional repression of interleukin- 6 gene by adenoviral E1A proteins. J. Biol. Chem. 267, 24886-24891. doi: 10.1016/S0021-9258(18)35846-0

Jang, H. J., and Song, K. D. (2020). Expression patterns of innate immunityrelated genes in response to polyinosinic:polycytidylic acid (poly[I:C]) stimulation in DF-1 chicken fibroblast cells. J. Anim. Sci. Technol. 62, 385-395. doi: $10.5187 /$ jast.2020.62.3.385

Jarosinski, K. W., Donovan, K. M., and Du, G. (2015). Expression of fluorescent proteins within the repeat long region of the Marek's disease virus genome allows direct identification of infected cells while retaining full pathogenicity. Virus Res. 201, 50-60. doi: 10.1016/j.virusres.2015.02.012

Jarosinski, K. W., Osterrieder, N., Nair, V. K., and Schat, K. A. (2005). Attenuation of Marek's disease virus by deletion of open reading frame RLORF4 but not RLORF5a. J. Virol. 79, 11647-11659. doi: 10.1128/ JVI.79.18.11647-11659.2005

Jarosinski, K. W., and Schat, K. A. (2007). Multiple alternative splicing to exons II and III of viral interleukin-8 (vIL-8) in the Marek's disease virus genome: the importance of vIL-8 exon I. Virus Genes 34, 9-22. doi: 10.1007/ s11262-006-0004-9

Keestra, A. M., De Zoete, M. R., Bouwman, L. I., and Van Putten, J. P. (2010). Chicken TLR21 is an innate CPG DNA receptor distinct from mammalian TLR9. J. Immunol. 185, 460-467. doi: 10.4049/jimmunol.0901921

Kim, M. S., Lim, T. H., Lee, D. H., Youn, H. N., Yuk, S. S., Kim, B. Y., et al. (2014). An inactivated oil-emulsion fowl adenovirus serotype 4 vaccine provides broad cross-protection against various serotypes of fowl adenovirus. Vaccine 32, 3564-3568. doi: 10.1016/j.vaccine.2014.03.015

Kim, T., Pazhoor, S., Bao, M., Zhang, Z., Hanabuchi, S., Facchinetti, V., et al. (2010). Aspartate-glutamate-alanine-histidine box motif (DEAH)/RNA helicase A helicases sense microbial DNA in human plasmacytoid dendritic cells. Proc. Natl. Acad. Sci. U. S. A. 107, 15181-15186. doi: 10.1073/ pnas. 1006539107

Kranzusch, P. J., Lee, A. S., Berger, J. M., and Doudna, J. A. (2013). Structure of human cGAS reveals a conserved family of second-messenger enzymes in innate immunity. Cell Rep. 3, 1362-1368. doi: 10.1016/j.celrep.2013.05.008

Kut, E., and Rasschaert, D. (2004). Assembly of Marek's disease virus (MDV) capsids using recombinant baculoviruses expressing MDV capsid proteins. J. Gen. Virol. 85, 769-774. doi: 10.1099/vir.0.19725-0

Laidlaw, S. M., Robey, R., Davies, M., Giotis, E. S., Ross, C., Buttigieg, K., et al. (2013). Genetic screen of a mutant poxvirus library identifies an ankyrin repeat protein involved in blocking induction of avian type I interferon. J. Virol. 87, 5041-5052. doi: 10.1128/JVI.02736-12

Li, K., Liu, Y., Xu, Z., Zhang, Y., Luo, D., Gao, Y., et al. (2019a). Avian oncogenic herpesvirus antagonizes the cGAS-STING DNA-sensing pathway to mediate immune evasion. PLoS Pathog. 15:e1007999. doi: 10.1371/journal. ppat.1007999

Li, X., Shu, C., Yi, G., Chaton, C. T., Shelton, C. L., Diao, J., et al. (2013a). Cyclic GMP-AMP synthase is activated by double-stranded DNA-induced oligomerization. Immunity 39, 1019-1031. doi: 10.1016/j.immuni.2013. 10.019

Li, Y., Wang, Y., Fang, L., Fu, J., Cui, S., Zhao, Y., et al. (2016). Genomic analysis of the chicken infectious anemia virus in a specific pathogen-free chicken population in China. Biomed. Res. Int. 2016:4275718. doi: $10.1155 / 2016 / 3514898$ 
Li, X. D., Wu, J., Gao, D., Wang, H., Sun, L., and Chen, Z. J. (2013b). Pivotal roles of cGAS-cGAMP signaling in antiviral defense and immune adjuvant effects. Science 341, 1390-1394. doi: 10.1126/science.1244040

Li, D., Wu, R., Guo, W., Xie, L., Qiao, Z., Chen, S., et al. (2019b). STINGmediated IFI16 degradation negatively controls type I interferon production. Cell Rep. 29, 1249.e1244-1260.e1244. doi: 10.1016/j.celrep.2019.09.069

Li, S., Yang, J., Zhu, Y., Ji, X., Wang, K., Jiang, S., et al. (2020). Chicken DNA sensing cGAS-STING signal pathway mediates broad spectrum antiviral functions. Vaccine 8:369. doi: 10.3390/vaccines 8030369

Li, P. H., Zheng, P. P., Zhang, T. F., Wen, G. Y., Shao, H. B., and Luo, Q. P. (2017). Fowl adenovirus serotype 4: epidemiology, pathogenesis, diagnostic detection, and vaccine strategies. Poult. Sci. 96, 2630-2640. doi: 10.3382/ ps/pex087

Liu, Y., Gao, L., Xu, Z., Luo, D., Zhang, Y., Gao, Y., et al. (2019). Marek's disease virus RLORF4 inhibits type I interferon production by antagonizing NF-kappaB activation. J. Virol. 93, e01037-e01119. doi: 10.1128/JVI.01037-19

Mansoor, M. K., Hussain, I., Arshad, M., and Muhammad, G. (2011). Preparation and evaluation of chicken embryo-adapted fowl adenovirus serotype 4 vaccine in broiler chickens. Trop. Anim. Health Prod. 43, 331-338. doi: 10.1007/ s11250-010-9694-z

McConnell, C. D., Adair, B. M., and Mcnulty, M. S. (1993). Effects of chicken anemia virus on macrophage function in chickens. Avian Dis. 37, 358-365. doi: $10.2307 / 1591659$

Miller, M. M., Jarosinski, K. W., and Schat, K. A. (2005). Positive and negative regulation of chicken anemia virus transcription. J. Virol. 79, 2859-2868. doi: 10.1128/JVI.79.5.2859-2868.2005

Neerukonda, S. N., and Katneni, U. (2020). Avian pattern recognition receptor sensing and signaling. Vet. Sci. 7:14. doi: 10.3390/vetsci7010014

Niu, Q., Cheng, Y., Wang, H., Yan, Y., and Sun, J. (2019a). Chicken DDX3X activates IFN-beta via the chSTING-chIRF7-IFN-beta signaling axis. Front. Immunol. 10:822. doi: 10.3389/fimmu.2019.00822

Niu, Y., Sun, Q., Shi, Y., Ding, Y., Li, Z., Sun, Y., et al. (2019b). Immunosuppressive potential of fowl adenovirus serotype 4. Poult. Sci. 98, 3514-3522. doi: $10.3382 / \mathrm{ps} / \mathrm{pez} 179$

$\mathrm{Ou}$, S. C., and Giambrone, J. J. (2012). Infectious laryngotracheitis virus in chickens. World J. Virol. 1, 142-149. doi: 10.5501/wjv.v1.i5.142

Ouyang, S., Song, X., Wang, Y., Ru, H., Shaw, N., Jiang, Y., et al. (2012). Structural analysis of the STING adaptor protein reveals a hydrophobic dimer interface and mode of cyclic di-GMP binding. Immunity 36, 1073-1086. doi: 10.1016/j.immuni.2012.03.019

Pan, Q., Yang, Y., Gao, Y., Qi, X., Liu, C., Zhang, Y., et al. (2017). An inactivated novel genotype fowl adenovirus 4 protects chickens against the hydropericardium syndrome that recently emerged in China. Viruses 9:216. doi: $10.3390 / \mathrm{v} 9080216$

Qi, Y., Yan, B., Chen, S., Chen, H., Wang, M., Jia, R., et al. (2016). CpG oligodeoxynucleotide-specific goose TLR21 initiates an anti-viral immune response against NGVEV but not AIV strain H9N2 infection. Immunobiology 221, 454-461. doi: 10.1016/j.imbio.2015.11.005

Qiao, X., Zong, Y., Liu, Z., Wu, Z., Li, Y., Wang, L., et al. (2021). The cGAS/ STING-TBK1-IRF regulatory Axis orchestrates a primitive interferon-Like antiviral mechanism in oyster. Front. Immunol. 12:689783. doi: 10.3389/ fimmu.2021.689783

Ran, J. S., Jin, J., Zhang, X. X., Wang, Y., Ren, P., Li, J. J., et al. (2018). Molecular characterization, expression and functional analysis of chicken sting. Int. J. Mol. Sci. 19:3706. doi: 10.3390/ijms19123706

Ren, G., Wang, H., Huang, M., Yan, Y., Liu, F., and Chen, R. (2019). Transcriptome analysis of fowl adenovirus serotype 4 infection in chickens. Virus Genes 55, 619-629. doi: 10.1007/s11262-019-01676-w

Rutz, M., Metzger, J., Gellert, T., Luppa, P., Lipford, G. B., Wagner, H., et al. (2004). Toll-like receptor 9 binds single-stranded CpG-DNA in a sequenceand pH-dependent manner. Eur. J. Immunol. 34, 2541-2550. doi: 10.1002/ eji.200425218

Sachan, S., Dhama, K., Latheef, S. K., Samad, H. A., Mariappan, A. K., Munuswamy, P., et al. (2019). Immunomodulatory potential of tinospora cordifolia and CpG ODN (TLR21 agonist) against the very virulent, infectious bursal disease virus in SPF chicks. Vaccine 7:106. doi: 10.3390/ vaccines 7030106

Sato, H., Oshiumi, H., Takaki, H., Hikono, H., and Seya, T. (2015). Evolution of the DEAD box helicase family in chicken: chickens have no DHX9 ortholog. Microbiol. Immunol. 59, 633-640. doi: 10.1111/1348-0421.12322

Schoggins, J. W., Macduff, D. A., Imanaka, N., Gainey, M. D., Shrestha, B., Eitson, J. L., et al. (2014). Pan-viral specificity of IFN-induced genes reveals new roles for cGAS in innate immunity. Nature 505, 691-695. doi: 10.1038/ nature 12862

Shah, M. S., Ashraf, A., Khan, M. I., Rahman, M., Habib, M., and Qureshi, J. A. (2016). Molecular cloning, expression and characterization of $100 \mathrm{~K}$ gene of fowl adenovirus-4 for prevention and control of hydropericardium syndrome. Biologicals 44, 19-23. doi: 10.1016/j.biologicals.2015.10.002

Shah, M. S., Ashraf, A., Rahman, M., Khan, M. I., and Qureshi, J. A. (2012). A subunit vaccine against hydropericardium syndrome using adenovirus penton capsid protein. Vaccine 30, 7153-7156. doi: 10.1016/j.vaccine.2012.10.013

Shang, G., Zhang, C., Chen, Z. J., Bai, X. C., and Zhang, X. (2019). Cryo-EM structures of STING reveal its mechanism of activation by cyclic GMP-AMP. Nature 567, 389-393. doi: 10.1038/s41586-019-0998-5

Shang, G., Zhu, D., Li, N., Zhang, J., Zhu, C., Lu, D., et al. (2012). Crystal structures of STING protein reveal basis for recognition of cyclic di-GMP. Nat. Struct. Mol. Biol. 19, 725-727. doi: 10.1038/nsmb.2332

Sharma, B. K., Kakker, N. K., Bhadouriya, S., and Chhabra, R. (2020). Effect of TLR agonist on infections bronchitis virus replication and cytokine expression in embryonated chicken eggs. Mol. Immunol. 120, 52-60. doi: 10.1016/j.molimm.2020.02.001

Skinner, M. A., Laidlaw, S. M., Eldaghayes, I., Kaiser, P., and Cottingham, M. G. (2005). Fowlpox virus as a recombinant vaccine vector for use in mammals and poultry. Expert Rev. Vaccines 4, 63-76. doi: 10.1586/14760584.4.1.63

Sun, L., Wu, J., Du, F., Chen, X., and Chen, Z. J. (2013). Cyclic GMP-AMP synthase is a cytosolic DNA sensor that activates the type I interferon pathway. Science 339, 786-791. doi: 10.1126/science.1232458

Sun, G. R., Zhou, L. Y., Zhang, Y. P., Zhang, F., Yu, Z. H., Pan, Q., et al. (2019). Differential expression of type I interferon mRNA and protein levels induced by virulent Marek's disease virus infection in chickens. Vet. Immunol. Immunopathol. 212, 15-22. doi: 10.1016/j.vetimm.2019.04.010

Trinh, D. Q., Ogawa, H., Bui, V. N., Baatartsogt, T., Kizito, M. K., Yamaguchi, S., et al. (2015). Characterization of $m A b s$ to chicken anemia virus and epitope mapping on its viral protein, VP1. J. Gen. Virol. 96, 1086-1097. doi: 10.1099/ vir.0.000042

Unterholzner, L., Keating, S. E., Baran, M., Horan, K. A., Jensen, S. B., Sharma, S., et al. (2010). IFI16 is an innate immune sensor for intracellular DNA. Nat. Immunol. 11, 997-1004. doi: 10.1038/ni.1932

Vega-Rodriguez, W., Ponnuraj, N., and Jarosinski, K. W. (2019). Marek's disease alphaherpesvirus (MDV) RLORF4 is not required for expression of glycoprotein $\mathrm{C}$ and interindividual spread. Virology 534, 108-113. doi: 10.1016/j. virol.2019.06.008

Vitak, N., Hume, D. A., Chappell, K. J., Sester, D. P., and Stacey, K. J. (2016). Induction of interferon and cell death in response to cytosolic DNA in chicken macrophages. Dev. Comp. Immunol. 59, 145-152. doi: 10.1016/j. dci.2016.01.023

Wang, J., Ba, G., Han, Y. Q., Ming, S. L., Wang, M. D., Fu, P. F., et al. (2020). Cyclic GMP-AMP synthase is essential for cytosolic double-stranded DNA and fowl adenovirus serotype 4 triggered innate immune responses in chickens. Int. J. Biol. Macromol. 146, 497-507. doi: 10.1016/j. ijbiomac.2020.01.015

Wang, J., Chu, B., Du, L., Han, Y., Zhang, X., Fan, S., et al. (2015). Molecular cloning and functional characterization of porcine cyclic GMP-AMP synthase. Mol. Immunol. 65, 436-445. doi: 10.1016/j.molimm.2015.02.002

Wang, L., Wen, M., and Cao, X. (2019). Nuclear hnRNPA2B1 initiates and amplifies the innate immune response to DNA viruses. Science 365:eaav0758. doi: $10.1126 /$ science.aav0758

Wang, Z., and Zhao, J. (2019). Pathogenesis of hypervirulent fowl adenovirus serotype 4: the contributions of viral and host factors. Viruses 11:741. doi: $10.3390 / \mathrm{v} 11080741$

Wei, T., Gong, J., Jamitzky, F., Heckl, W. M., Stark, R. W., and Rossle, S. C. (2009). Homology modeling of human toll-like receptors TLR7, 8, and 9 ligand-binding domains. Protein Sci. 18, 1684-1691. doi: 10.1002/pro.186

Zhang, C., Shang, G., Gui, X., Zhang, X., Bai, X. C., and Chen, Z. J. (2019). Structural basis of STING binding with and phosphorylation by TBK1. Nature 567, 394-398. doi: 10.1038/s41586-019-1000-2

Zhang, X., Shi, H., Wu, J., Zhang, X., Sun, L., Chen, C., et al. (2013). Cyclic GMP-AMP containing mixed phosphodiester linkages is an endogenous 
high-affinity ligand for STING. Mol. Cell 51, 226-235. doi: 10.1016/j. molcel.2013.05.022

Zhang, Z., Yuan, B., Bao, M., Lu, N., Kim, T., and Liu, Y. J. (2011). The helicase DDX41 senses intracellular DNA mediated by the adaptor STING in dendritic cells. Nat. Immunol. 12, 959-965. doi: 10.1038/ni.2091

Zhao, J., Zhong, Q., Zhao, Y., Hu, Y. X., and Zhang, G. Z. (2015). Pathogenicity and complete genome characterization of fowl adenoviruses isolated from chickens associated with inclusion body hepatitis and hydropericardium syndrome in China. PLoS One 10:e0133073. doi: 10.1371/journal.pone. 0145646

Zhong, B., Yang, Y., Li, S., Wang, Y. Y., Li, Y., Diao, F., et al. (2008). The adaptor protein MITA links virus-sensing receptors to IRF3 transcription factor activation. Immunity 29, 538-550. doi: 10.1016/j.immuni.2008. 09.003

Zsak, L., and Kisary, J. (1984). Grouping of fowl adenoviruses based upon the restriction patterns of DNA generated by BamHI and HindIII. Intervirology 22, 110-114. doi: 10.1159/000149541
Conflict of Interest: The authors declare that the research was conducted in the absence of any commercial or financial relationships that could be construed as a potential conflict of interest.

Publisher's Note: All claims expressed in this article are solely those of the authors and do not necessarily represent those of their affiliated organizations, or those of the publisher, the editors and the reviewers. Any product that may be evaluated in this article, or claim that may be made by its manufacturer, is not guaranteed or endorsed by the publisher.

Copyright (c) 2021 Gao, Zheng and Wang. This is an open-access article distributed under the terms of the Creative Commons Attribution License (CC BY). The use, distribution or reproduction in other forums is permitted, provided the original author(s) and the copyright owner(s) are credited and that the original publication in this journal is cited, in accordance with accepted academic practice. No use, distribution or reproduction is permitted which does not comply with these terms. 